\title{
Dicaine represses apoptosis-linked gene 2-interacting protein $X$ expression to induce airway epithelial barrier dysfunction
}

\author{
JING-LI CHEN and HONG YAN \\ Department of Anesthesiology, Wuhan Central Hospital, Wuhan, Hubei 430014, P.R. China \\ Received May 26, 2014; Accepted February 11, 2015
}

DOI: $10.3892 / \mathrm{mmr} .2015 .3433$

\begin{abstract}
Epithelial barrier dysfunction is associated with a number of inflammatory disorders. However, the pathogenesis of epithelial barrier dysfunction is unclear. This study aims to elucidate the involvement of dicaine in airway epithelial barrier dysfunction. In the present study, an RPMI2650 (Rpc) human airway epithelial cell line was cultured, with or without dicaine, in monolayers using Transwells. In order to assess airway epithelial barrier function, the levels of transepithelial electrical resistance and permeability to ovalbumin (OVA) were measured. Expression of apoptosis-linked gene 2-interacting protein $\mathrm{X}$ (Alix) in Rpc cells was assessed using quantitative reverse transcription-polymerase chain reaction and western blotting. The antigenicity of OVA was assessed using a $\mathrm{T}$ cell proliferation assay. The results of the present study demonstrated that Alix expression levels were markedly lower in Rpc cells treated with dicaine, compared with those not treated with dicaine. An increase in the level of transcellular permeability to OVA was observed in Rpc monolayers following treatment with dicaine, compared with that in the Rpc monolayer without dicaine treatment. Furthermore, the Rpc monolayer maintained high antigenicity and induced antigen specific $\mathrm{T}$ cell proliferation. In conclusion, dicaine causes a decrease in expression levels of Alix, which resulted in compromise of the Rpc cell monolayer epithelial barrier function.
\end{abstract}

\section{Introduction}

The epithelial barrier is important in maintaining homeostasis. Epithelial barrier dysfunction is associated with the pathogenesis of a number of inflammatory diseases, such as allergic asthma (1), intestinal allergy (2), inflammatory bowel diseases (3) and allergic dermatitis (4). The causes of epithelial barrier dysfunction are not fully understood.

Correspondence to: Dr Hong Yan, Department of Anesthesiology, Wuhan Central Hospital, 26 Shenli Street, Wuhan, Hubei 430014, P.R. China

E-mail: yhmzk1@126.com

Key words: Alix, dicaine, epithelial barrier, T cells, antigenicity
The epithelial layer serves as a barrier between the deep tissue and the external environment. Water and a number of small molecules, such as nutrients, are able to permeate the epithelial layer. However, large molecular substances, such as proteins with strong antigenicity, are prevented from permeating the epithelial barrier. A number of factors are associated with epithelial barrier dysfunction, such as psychological stress (5), neural factors (6) and thermal events (7).

Two permeability pathways that are associated with epithelial barrier function have been identified. In the paracellular pathway, substances permeate the paracellular space, and in the transcellular pathway, substances are endocytosed into the cytoplasm and degraded by enzymes. A number of factors may affect the endocytic substance in the cytoplasm. For example, psychological stress may result in incomplete degradation of endocytic antigens in the cytoplasm. Subsequently, these antigens may induce aberrant immune responses, which result in immune disorders (8).

Dicaine is used as a local anesthetic during eye and upper airway surgery. Previous reports have suggested that a number of local anesthetics may exhibit cytotoxic effects on epithelial cells (9), although whether dicaine affects epithelial barrier function, remains to be investigated. Apoptosis-linked gene 2 (ALG-2)-interacting protein $\mathrm{X}$ (Alix) is a ubiquitous adaptor protein initially described for its capacity to bind to the calcium-binding protein, ALG-2. ALG-2 is involved in apoptosis initiation (10) and protein-protein binding (11), and it may facilitate infection by human immunodeficiency virus (12). Decreases in Alix expression levels may be involved in the pathogenesis of colitis (13). The results of a previous study suggested that Alix is associated with epithelial barrier function (14). It is, therefore, hypothesized that Alix expression enables the maintenance of healthy epithelial barrier function.

\section{Materials and methods}

Reagents. Antibodies (H-270, polyclonal; 1:300) and an Alix small hairpin RNA (shRNA) kit were purchased from Santa Cruz Biotechnology Inc. (Shanghai, China). Reagents for quantitative reverse transcription-polymerase chain reaction (qRT-PCR) were purchased from Invitrogen Life Technologies (Shanghai, China). Ovalbumin (OVA) was purchased from Sigma-Aldrich (Shanghai, China). An OVA ELISA kit was purchased from Antibodies Online (Atlanta, GA, USA). Molecular cloning reagents were purchased from Invitrogen 
Life Technologies. The immune cell isolation kits were purchased from Miltenyi Biotec, Inc. (Shanghai, China).

Mice. A total of 24 DO11.10 mice (8-10 weeks old) were purchased from the Xian Experimental Animal Center (Xian, China). Mice were maintained in a pathogen-free environment. The experimental procedures using mice were approved by the Ethics Committee of Wuhan Central Hospital (Wuhan, China).

Cell culture. The RPMI 2650 human airway epithelial cell line $(\mathrm{Rpc})$ was obtained from the American Type Culture Collection (ATCC, Manassas, VA, USA). Rpc cells were cultured in Dulbecco's modified Eagle's medium (DMEM; Sigma-Aldrich), supplemented with $10 \%$ fetal bovine serum (Sigma-Aldrich), $100 \mathrm{U} / \mathrm{ml}$ penicillin, $0.1 \mathrm{mg} / \mathrm{ml}$ streptomycin and $200 \mathrm{mM} \mathrm{L-glutamine}$ at $37^{\circ} \mathrm{C}$ at $5 \% \mathrm{CO}_{2}$ (Sigma-Aldrich). Cells were seeded in Transwells $(0.4 \mu \mathrm{m}$ pore size; Corning Costar, Corning, NY, USA). Once the cells had reaching confluence, Rpc cell monolayers were used for subsequent experiments.

$q R T-P C R$. Alix mRNA expression was analyzed using qRT-PCR. Total RNA was extracted using TRIzol ${ }^{\circledR}$ reagent (Invitrogen Life Technologies). cDNA was synthesized using a reverse transcription kit (Invitrogen Life Technologies). $\beta$-actin was used as a standard. The following primer sequences were used: Forward: AAGGAACGTTGGCAAAGGAC and reverse: GAAGGGATGGCAGCATTCAG for Alix (AF151793), and forward: CGCAAAGACCTGTATGCCAA and reverse: CACACAGAGTACTTGCGCTC for $\beta$-actin (HQ154074). qRT-PCR was performed using a MiniOpticon qPCR system (Bio-Rad Laboratories, Shanghai, China) with the SYBR Green Master mix (Invitrogen Life Technologies). The cycling conditions included $10 \mathrm{~min}$ at $95^{\circ} \mathrm{C}$ followed by 40 cycles at $95^{\circ} \mathrm{C}$ for $15 \mathrm{sec}, 60^{\circ} \mathrm{C}$ for $30 \mathrm{sec}$ and $72^{\circ} \mathrm{C}$ for $30 \mathrm{sec}$. The $2^{-\Delta \Delta \mathrm{Ct}}$ method was used to calculate changes in Alix gene expression relative to that of $\beta$-actin.

Western blot analysis. Protein extracts $(50 \mu \mathrm{g})$ from the Rpc cells were dissolved in a sample buffer, heated for 5 min, separated on a $10 \%$ sodium dodecyl sulfate (SDS) polyacrylamide gel (Invitrogen Life Technologies) and then transferred onto a nitrocellulose membrane (Invitrogen Life Technologies). The blots were blocked for $1 \mathrm{~h}$ in Tris-buffered saline with $0.05 \%$ Tween- $20^{\circledR}$ (TBST; Invitrogen Life Technologies), containing 5\% non-fat milk, at room temperature, and washed three times with TBST. Subsequently, they were incubated at room temperature for $1 \mathrm{~h}$ with an anti-Alix antibody in TBST, containing 5\% non-fat dried milk. The blots were then washed three times with TBST and incubated for $1 \mathrm{~h}$ with horseradish peroxidase-conjugated rabbit antimouse Immunoglobulin G (cat. no. sc-358920; 1:5,000), at room temperature. After washing three times with TBST, they were visualized using an enhanced chemiluminescence detection system (Invitrogen Life Technologies). The results were recorded using X-ray films. The integrated density of the immune blots was assessed and quantified using Image $\mathrm{J}$ software (version 5.1; National Institutes of Health, Bethesda, MD, USA).
Transepithelial electric resistance (TER). The TER of the Rpc cell monolayers was measured using the Millicell ERS apparatus (EMD Millipore, Billerica, MA, USA) at 0 and $24 \mathrm{~h}$ after the addition of dicaine.

Assessment of epithelial monolayer permeability. Rpc cells were cultured for 2 weeks in Transwell inserts, until they reached confluence (TER $>100 \mathrm{ohm} / \mathrm{cm}^{2}$ ). Subsequently, OVA $(10 \mu \mathrm{g} / \mathrm{ml})$ was added to the inserts. Samples were removed from the basal chambers $24 \mathrm{~h}$ later and OVA levels were determined using ELISA.

ELISA. The concentration of OVA in the culture supernatant was determined by ELISA using a commercial reagent kit according to the manufacturer's instructions.

Alix RNA interference (RNAi). The Alix gene was knocked down in Rpc cells using an Alix-specific shRNA and a nonspecific shRNA was used as a positive control, according to the manufacturer's instructions. Lentiviral vectors carrying the shRNA of Alix or control vectors were added to the inserts.

Overexpression of Alix. Genomic DNA was extracted from Rpc cells, and the Alix gene was amplified by PCR. The PCR product was then cloned into the $\mathrm{pTZ57R/T}$ vector and transformed into Escherichia coli (TG1 strain; Invitrogen Life Technologies).

In order to subclone Alix into the pcDNA3 plasmid, the gene was cloned with linkers to join it to the HindIII and EcoRI sites of pcDNA3, in order to produce the recombinant eukaryotic expression plasmid, pcAlix. The upstream primer for the thiol-specific antioxidant antigen gene contains a HindIII site and the start codon, ATG. The downstream primer contains an EcoRI site and the stop codon, TAA. Competent E. coli cells (TG1 strain) were transformed using a ligation mixture (Invitrogen Life Technologies) and heat shock by placing the bottom half of the tube into a $42^{\circ} \mathrm{C}$ water bath for $45 \mathrm{sec}$. The plasmid was purified using a plasmid extraction kit (Invitrogen Life Technologies) according to the manufacturer's instructions and then sequenced. The purified PCR products were sequenced using the BigDye Terminator kit (Invitrogen Life Technologies) and separated by capillary electrophoresis (Sequencer model 3730XL; Applied Biosystems, Foster City, CA, USA). The plasmid recovered from the recombinant bacterial colony was sequenced by Jier Biotech (Guangzhou, China), in order to confirm the presence of the Alix gene.

$\mathrm{Rpc}$ cells were grown to $60-70 \%$ confluence in $5 \% \mathrm{CO}_{2}$ in DMEM, at $37^{\circ} \mathrm{C}$. The cells were washed using a serum-free medium. Transfection was performed using a transfection kit (Invitrogen Life Technologies) according to the manufacturer's instructions. The cells were incubated overnight, at $37^{\circ} \mathrm{C}$. Serum-free DMEM medium was mixed with the Genejuice reagent (Invitrogen Life Technologies) and incubated for $10 \mathrm{~min}$ at $37^{\circ} \mathrm{C}$. Subsequently, the recombinant plasmid was added to the mixture and incubated for $15 \mathrm{~min}$ at $37^{\circ} \mathrm{C}$. FCS (Sigma-Aldrich) was added to the DMEM medium, which was then added to the cells and incubated overnight, at $37^{\circ} \mathrm{C}$.

Isolation of immune cells. Dendritic cells (DCs) and CD4 ${ }^{+}$ CD25- $\mathrm{T}$ effector cells (Teff cells) were isolated from DO11.10 

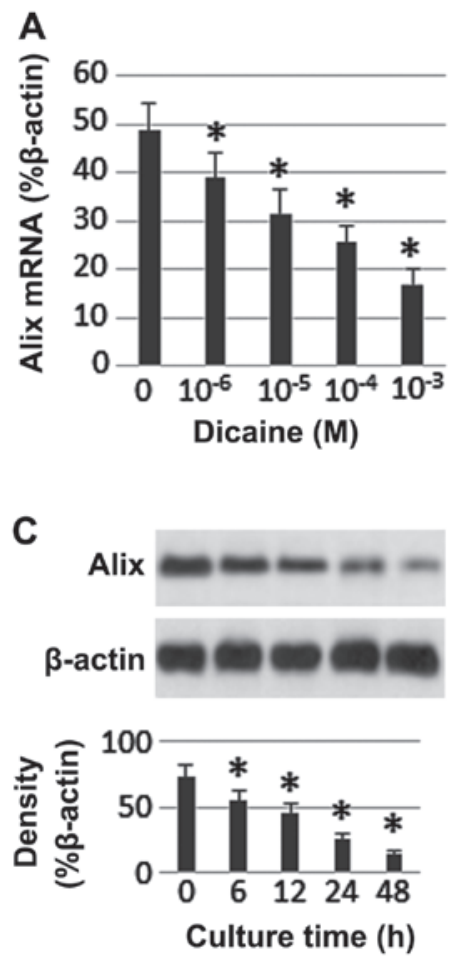
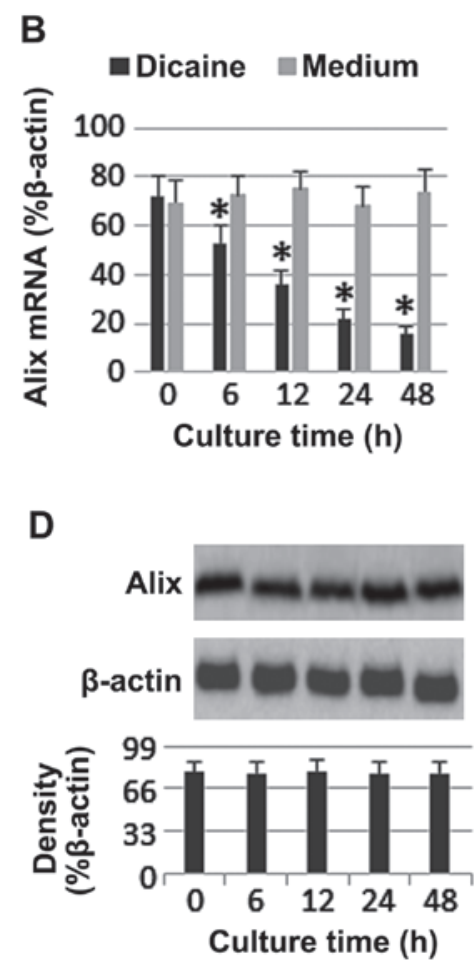

Figure 1. Dicaine suppresses Alix expression in Rpc cells. Rpc cells were exposed to dicaine at different concentrations for $48 \mathrm{~h}$ (A), or exposed to dicaine at $10^{-3} \mathrm{M}$ for $0-48 \mathrm{~h}(\mathrm{~B})$. Western blotting indicated the protein contents of Alix in Rpcs treated with dicaine (C) or control (D). Bars below the blots indicate the integrated density of the blots. Data are presented as the mean \pm standard deviation. ${ }^{*} \mathrm{P}<0.01$, compared with the control. The data represent three separate experiments. Alix, apoptosis-linked gene 2-interacting protein X; Rpc cells, RPMI2650 human airway epithelial cell line.

A

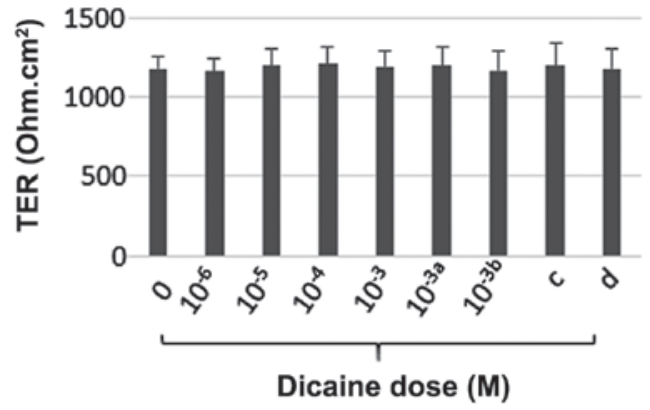

C

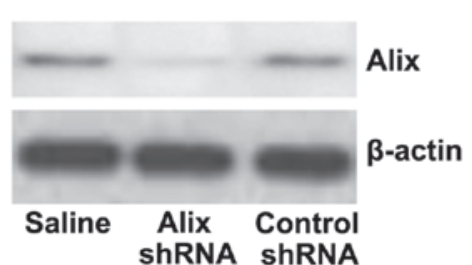

B

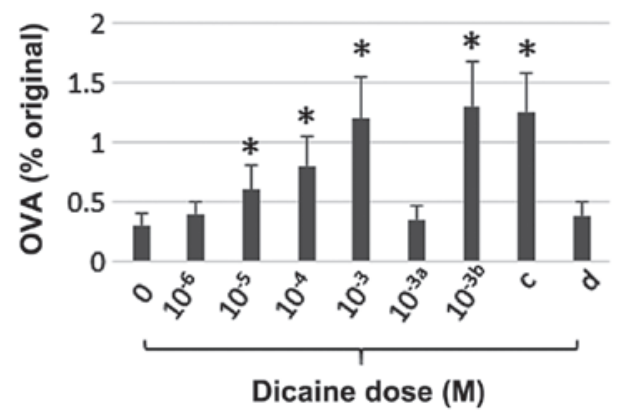

D

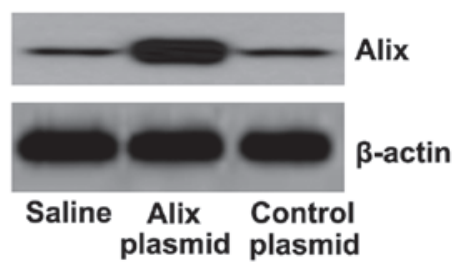

Figure 2. Assessment of Rpc cell monolayer barrier function. Rpc cell monolayers were treated with dicaine in Transwells with at different concentrations for $24 \mathrm{~h}$. (A) TER of Rpc monolayers. (B) Levels of OVA in the basal chambers of transwells. a, Alix-knockdown Rpc monolayers; b, rpc monolayers treated with control shRNA; c, rpcs with Alix overexpression; $d$, rpcs treated with control plasmids. "P<0.01, compared with group 0. (C) Alix gene. (D) Alix overexpression. The data represent three separate experiments. Rpc cells, RPMI2650 human airway epithelial cell line; Alix, apoptosis-linked gene 2-interacting protein X; TER, transepithelial electric resistance; OVA, ovalbumin; shRNA, small hairpin RNA.

mouse spleen using commercial reagent kits (Miltenyi Biotec, Shanghai, China), according to the manufacturer's instructions. Isolated cells were analyzed using flow cytometry; the level of purity was $>96 \%$. The cells were then cultured in RPMI-1640 medium (Sigma-Aldrich) for subsequent experiments.
Assessment of Teff cell proliferation. Isolated OVA-specific Teff cells [labeled with carboxyfluorescein diacetatesuccinimidyl ester (CFSE)] were cultured, for 3 days, in the presence of DCs (T cell:DC ratio = 5:1), in the culture supernatant collected from the basal chambers of transwells (containing 


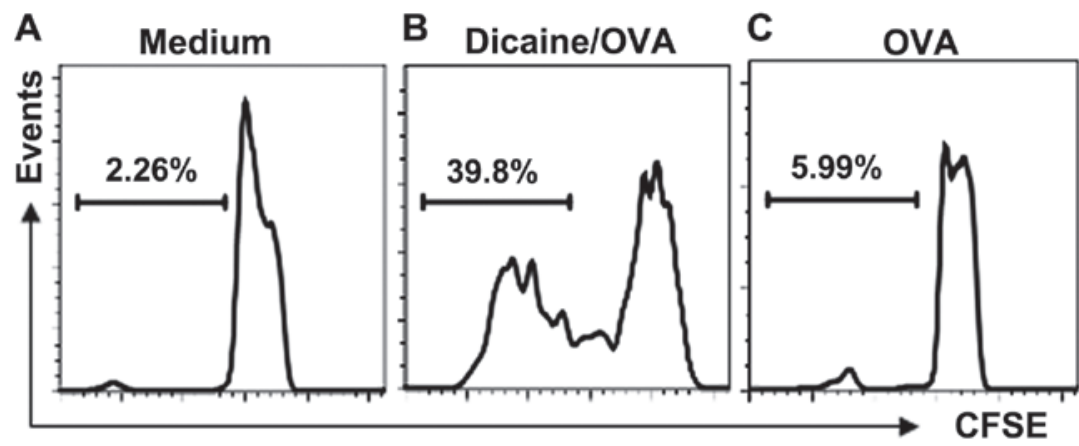

D

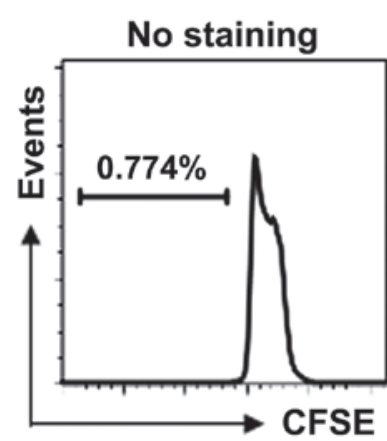

E

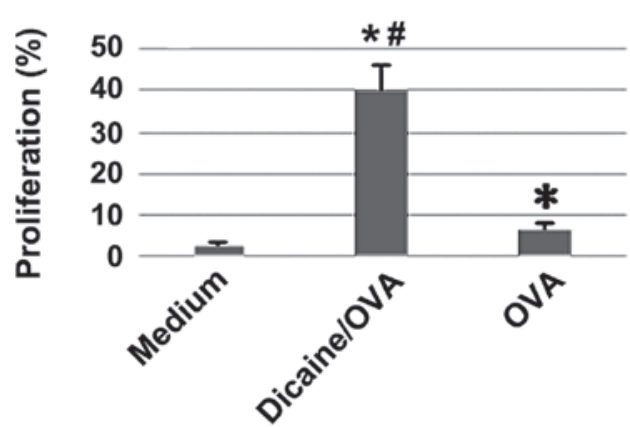

Figure 3. Assessment of T cell proliferation. The OVA-specific Teff cells were cultured for 3 days with the supernatant collected from the basal chambers of the Transwells. The treatment is indicated above the histogram panels. (A) Cells were treated with Dulbecco's modified Eagle's medium. (B) Cells were treated with dicaine and OVA. (C) Cells were treated with OVA alone. (D) Negative staining control. (A-D) Histograms indicate Teff proliferation. (E) Summarized data from (A-C). Data are presented as the mean \pm standard deviation. ${ }^{~} \mathrm{P}<0.01$, compared with the control and ${ }^{~} \mathrm{P}<0.01$, compared with the OVA group. The data represent three separate experiments. CFSE, carboxyfluorescein diacetatesuccinimidyl ester; OVA, ovalbumin; Teff, T effector cells.

OVA transported across the Rpc monolayers). Cells were then collected and analyzed using flow cytometry.

Statistical analysis. The data are presented as the mean \pm standard deviation. Differences between groups were assessed using analysis of variance. $\mathrm{P}<0.05$ was considered to indicate a statistically significant difference.

\section{Results}

Dicaine suppresses Alix expression in airway epithelial cells. In the present study, the effect of dicaine on the expression of Alix in Rpc cells was assessed. Alix mRNA and protein expression was detected in the Rpc cells. Dicaine treatment led to a decrease in Alix expression levels in a time-dependent manner (Fig. 1).

Alix is associated with the dicaine-induced upregulation of airway epithelial barrier permeability. The effects of dicaine and Alix expression on epithelial barrier functions were assessed. Rpc cell monolayers were treated with different concentrations of dicaine for $24 \mathrm{~h}$. The results suggested that treatment with dicaine did not affect the TER in the Rpc monolayers. However, treatment with dicaine led to an increase in the level of permeability to OVA in the Rpc monolayers, in a dose-dependent manner. Alix expression was subsequently knocked down in the Rpc cells, using RNAi. Following Alix expression knock-down, no difference was observed between the level of permeability to OVA in the Rpc monolayers treated with dicaine, compared with that in the control monolayers. By contrast, overexpression of Alix led to a marked increase in the level of permeability to OVA, and no difference in TER, in Rpc monolayers treated with Rpc, compared with the control monolayers, (Fig. 2). The results of the present study indicated that Alix expression may increase the transcellular permeability in Rpc monolayers.

Dicaine compromises antigen degradation by airwayepithelial cells. The antigenicity of OVA in dicaine-treated Rpc monolayers was measured. Teff cells were isolated from DO11.10 mouse spleens. In the dicaine-treated group 34.3\% Teff cell proliferation was observed, whereas in the control group only $6.5 \%$ Teff cell proliferation was observed $(\mathrm{P}<0.01$; Fig. 3 ). These results indicated that antigens maintained strong antigenicity and were more capable of permeating the dicaine-treated Rpc monolayers, compared with monolayers that had not been treated with dicaine.

\section{Discussion}

The results of the present study suggest that dicaine, a local anesthetic, may compromise epithelial barrier functions. Using Rpc monolayers as an in vitro epithelial barrier model, the current study demonstrated that dicaine treatment may lead to an increase in Alix expression in Rpc cells. Treatment with dicaine caused an increase in the transcellular permeability of the Rpc monolayers, and this effect was reversed following Alix gene knock-down. Alix gene overexpression compromised epithelial barrier function and led to an increase in the capability of antigens to permeate the Rpc monolayer 
barrier. These antigens were shown to induce antigen-specific T cell proliferation.

Epithelial barrier dysfunction is a common pathological condition that is observed in a number of types of inflammatory disorders. However, the pathogenesis of epithelial barrier dysfunction remains poorly understood. Yang et al (15) reported that $\mathrm{CD} 23$ expression leads to an increase in specific antigen transcellular transport across the epithelial barrier, which interferes with the efficiency of antigen degradation in the cytoplasm $(16,17)$. The results of the present study are in accordance with previous studies $(18,19)$ and suggest that dicaine may affect the epithelial barrier function by increasing the transcellular transport. Yang et al (18) demonstrated that stress-induced intestinal epithelial barrier dysfunction induced typical intestinal $\mathrm{T}$ helper 2 type inflammation. Saatian et al (19) indicated that Th2-cytokine expression induces epithelial barrier dysfunction, which may contribute to airway inflammation in allergic asthma.

In the present study, dicaine treatment led to the upregulation of Alix expression in Rpc cells. Alix expression has multiple functions, such as facilitating apoptosis by activating caspase 9 (10) and interacting with endosomal sorting complexes required for transport (20). The results of the present study indicated that dicaine treatment leads to an increase in the expression of Alix in Rpc cells, which increases the level of transcellular permeability. The antigens transported across the Rpc monolayers maintained strong levels of antigenicity. This observation indicates that the endocytic antigens were not degraded after permeating the Rpc cytoplasm. Alix overexpression induces caspase activation and cell apoptosis (21). Furthermore, Alix expression is involved in the endolysosomal system, including in regulating endosomal trafficking (21). The results of the present study, therefore, suggest that Alix expression may compromise the process of endocytic antigen degradation within the cytoplasm. The mechanisms underlying this process require further investigation.

In conclusion, the present study demonstrates that dicaine treatment increases the expression of Alix in Rpc cells. Alix expression increases the transcellular permeability of Rpc monolayers. The transported antigens maintain their antigenicity.

\section{References}

1. Leino MS, Loxham M, Blume C, et al: Barrier disrupting effects of alternaria alternata extract on bronchial epithelium from asthmatic donors. PLoS One 8: e71278, 2013.

2. Suzuki T: Regulation of intestinal epithelial permeability by tight junctions. Cell Mol Life Sci 70: 631-659, 2013.

3. McCole DF: Regulation of epithelial barrier function by the inflammatory bowel disease candidate gene, PTPN2. Ann NY Acad Sci 1257: 108-114, 2012.
4. Sevilla LM, Latorre V, Sanchis A and Pérez, P: Epidermal inactivation of the glucocorticoid receptor triggers skin barrier defects and cutaneous inflammation. J Invest Dermatol 133: 361-370, 2013.

5. Yu Y,Liu ZQ, Liu XY, et al: Stress-derived corticotropin releasing factor breaches epithelial endotoxin tolerance. PLoS One 8: e65760, 2013.

6. Zheng PY, Feng BS, Oluwole C, et al: Psychological stress induces eosinophils to produce corticotrophin releasing hormone in the intestine. Gut 58: 1473-1479, 2009.

7. Liu T, Wang BQ, Wang CS and Yang PC: Concurrent exposure to thermal stress and oral Ag induces intestinal sensitization in the mouse by a mechanism of regulation of IL-12 expression. Immunol Cell Biol 84: 430-439, 2006.

8. Yang PC, Jury J, Söderholm JD, et al: Chronic psychological stress in rats induces intestinal sensitization to luminal antigens. Am J Pathol 168: 104-114, 2006.

9. Kobayashi K, Ohno S, Uchida S, Amano O, Sakagami H and Nagasaka H: Cytotoxicity and type of cell death induced by local anesthetics in human oral normal and tumor cells. Anticancer Res 32: 2925-2933, 2012.

10. Strappazzon F, Torch S, Chatellard-Causse C, et al: Alix is involved in caspase 9 activation during calcium-induced apoptosis. Biochem Biophys Res Commun 397: 64-69, 2010.

11. Fisher RD, Chung HY, Zhai Q, et al: Structural and biochemical studies of ALIX/AIP1 and its role in retrovirus budding. Cell 128: 841-852, 2007.

12. Zhai Q, Fisher RD, Chung HY, et al: Structural and functional studies of ALIX interactions with YPXnL late domains of HIV-1 and EIAV. Nat Struct Mol Biol 15: 43-49, 2008.

13. Hoffmann M, Kim SC, Sartor RB and Haller D: Enterococcus faecalis strains differentially regulate Alix/AIP1 protein expression and ERK 1/2 activation in intestinal epithelial cells in the context of chronic experimental colitis. J Proteome Res 8: 1183-1192, 2009.

14. Yan H, Yi H, Xia L, et al: Staphylococcal enterotoxin B suppresses Alix and compromises intestinal epithelial barrier functions. J Biomed Sci 21: 29, 2014.

15. Yang PC, Berin MC, Yu LC, et al: Enhanced intestinal transepithelial antigen transport in allergic rats is mediated by $\operatorname{IgE}$ and CD23 (FcepsilonRII). J Clin Invest 106: 879-886, 2000

16. Yu LC, Yang PC, Berin MC, et al: Enhanced transepithelial antigen transport in intestine of allergic mice is mediated by IgE/CD23 and regulated by interleukin-4. Gastroenterology 121: 370-381, 2001.

17. Yu LC, Montagnac G, Yang PC, et al: Intestinal epithelial CD23 mediates enhanced antigen transport in allergy: evidence for novel splice forms. Am J Physiol Gastrointest Liver Physiol 285: G223-G234, 2003

18. Yang PC, Jury J, Söderholm JD, Sherman PM, McKay DM and Perdue MH: Chronic psychological stress in rats induces intestinal sensitization to luminal antigens. Am J Pathol 168: 104-114, 2006.

19. Saatian B, Rezaee F, Desando S, Emo J, Chapman T, Knowlden S and Georas SN: Interleukin-4 and interleukin-13 cause barrier dysfunction in human airway epithelial cells. Tissue Barriers 1: e24333, 2013.

20. Mahul-Mellier AL, Strappazzon F, Chatellard-Causse C, Blot B, Béal D, Torch S, Hemming F, Petiot A, Verna JM, Fraboulet S and Sadoul R: Alix and ALG-2 make a link between endosomes and neuronal death. Biochem Soc Trans 37: 200-203, 2009.

21. Trioulier Y, Torch S, Blot B, Cristina N, Chatellard-Causse C, Verna JM and Sadoul R: Alix, a protein regulating endosomal trafficking, is involved in neuronal death. J Biol Chem 279: 2046-2052, 2004. 